\title{
ROTATIONAL-TRANSLATIONAL ADDITION THEOREMS FOR SCALAR SPHEROIDAL WAVE FUNCTIONS*
}

BY

\author{
R. H. MACPHIE, J. DALMAS, AND R. DELEUIL \\ Université de Provence, Marseille, France
}

\begin{abstract}
Rotational-translational addition theorems for the scalar spheroidal wave function $\psi_{m n}^{(i)}(h ; \eta, \xi, \phi)$, with $i=1,3,4$, are deduced. This permits one to represent the $m n^{\text {th }}$ scalar spheroidal wave function, associated with one spheroidal coordinate system $\left(h_{q} ; \eta_{q}, \xi_{q}, \phi_{q}\right)$, centered at its local origin $O_{q}$, by an addition series of spheroidal wave functions associated with a second rotated and translated system $\left(h_{r} ; \eta_{r}, \xi_{r}, \phi_{r}\right)$, centered at $O_{r}$. Such theorems are necessary in the rigorous analysis of radiation and scattering by spheroids with arbitrary spacings and orientations.
\end{abstract}

1. Introduction. The formally exact eigenfunction solution to the problem of acoustic or electromagnetic scattering of plane waves by two or more spherical or spheroidal scatterers requires a knowledge of certain addition theorems. About a quarter of a century ago scalar translational addition theorems for spherical wave functions were presented by Friedman and Russek [1]; Stein [2] and Cruzan [3] later gave us the corresponding theorems for the vector (electromagnetic) case. Moreover, Stein [2, pp. 17 and 20-21] also described addition theorems for the case of rigid body rotations in spherical coordinates. The vector translational theorems were successfully applied to the problem of electromagnetic scattering by two spheres by Bruning and Lo [4].

More recently, Sinha and MacPhie [5] obtained translational addition theorems for both scalar and vector spheroidal wave functions, with the vector function $\mathbf{M}_{m n}^{(a)}$ given in terms of the scalar function $\Psi_{m n}$ by the generating operator $\mathbf{M}_{m n}^{(a)}=\nabla \Psi_{m n} \times \hat{a}$, where $\hat{a}=\hat{x}, \hat{y}$, $\hat{z}$. The alternative formulation, where $\hat{a}$ is given by the radius vector $\mathbf{r}$, has resulted in the translational addition theorems for $\mathbf{M}_{m n}^{(r)}$ and $\mathbf{N}_{m n}^{(r)}=k^{-1} \nabla \times \mathbf{M}_{m n}^{(r)}$ due to Dalmas and Deleuil $[6$, Sec. $2 ; 7]$. These theorems have found application in the problem of electromagnetic scattering of a plane wave from a pair of perfectly conducting prolate spheroids whose major axes are in parallel alignment $[6,8,9]$. Moreover, very thin conducting spheroids can be used to model thin-wire dipole antennas, and translational addition

\footnotetext{
${ }^{*}$ Received June 26, 1985.
} 
theorems have recently been used to deduce the mutual admittance of spheroidal dipole antennas in parallel configuration [10].

In this paper we consider the more general scattering problem in which the major axis of each spheroid is arbitrarily oriented. Such configurations serve as models in such practical disciplines as radar scattering from complex targets, microwave heating of complicated samples, and biomedical engineering.

Section 2 treats the quite simple problem of obtaining an addition theorem for the case of scalar wave functions when one spheroidal coordinate system is transformed into another by a simple rigid body rotation. The rotation is described by means of the well-known Euler angles $(\alpha, \beta, \gamma)$. In Sec. 3 the rotational addition theorem of Sec. 2 is coupled with the translational addition theorem previously obtained for scalar wave functions to deduce the required scalar rotational-translational addition theorem in which the $m n^{\text {th }}$ scalar wave function $\Psi_{m n}^{(i)}\left(h_{q} ; \mathbf{r}_{q}\right)$, associated with the $q^{\text {th }}$ spheroidal coordinate system, is given by a series expansion in terms of the spheroidal scalar wave function $\Psi_{\mu \nu}^{(j)}\left(h_{r} ; \mathbf{r}_{r}\right)$ of the $r^{\text {th }}$ spheroidal coordinate system. The coordinates $(\eta, \xi, \phi)$ and notations are those given by Flammer [11], and the reader not familiar with such matters is referred to this work. However, in the present paper $h=k F$, where $k$ is the wave number of the assumed harmonic radiation and $F$ is the semi-interfocal distance of the spheroids.

In Section 4 it is shown that the coefficient of $\Psi_{\mu \nu}^{(j)}\left(h_{r} ; \mathbf{r}_{r}\right)$ in the series expansion of $\Psi_{m n}^{(i)}\left(h_{q} ; \mathbf{r}_{q}\right)$ can be represented in terms of a matrix product, each factor of which can be identified with a step, e.g., rotation or translation, in the overall transformation. Section 5 concludes the work with a discussion of the related problem of obtaining rotational-translational theorems for the vector case.

2. Rotational addition theorem for scalar spheroidal wave functions. We consider, as illustrated in Fig. 1, two Cartesian coordinate systems which share a common origin $O$. The unprimed system $(x, y, z)$ is, by rigid body rotation, transformable into the primed system $\left(x^{\prime}, y^{\prime}, z^{\prime}\right)$ by means of rotations through the Euler angles $\alpha, \beta, \gamma$, as described by Edmonds [12]. Our task is the relatively simple one of transforming the scalar spheroidal wave function $\Psi_{m n}^{(i)}(h ; \eta, \xi, \phi)$, having coordinates whose axis of symmetry is the $z$-axis of the unprimed Cartesian system $(x, y, z)$, into an addition series expansion of spheroidal scalar wave functions $\Psi_{\mu \nu}^{\prime(i)}\left(h^{\prime} ; \eta^{\prime}, \xi^{\prime}, \phi^{\prime}\right)$, whose symmetry axis is the $z^{\prime}$-axis of the primed system $\left(x^{\prime}, y^{\prime}, z^{\prime}\right)$.

We begin with an integral representation [11, p. 48; 5, p. 145] of the $m n^{\text {th }}$ scalar spheroidal wave function in the unprimed system, in particular, that which is of the first kind, representable in the radial $(\xi)$ direction by a series expansion of Bessel functions of the first kind:

$$
\Psi_{m n}^{(1)}(h ; \eta, \xi, \phi)=\frac{1}{4 \pi j^{n}} \int_{0}^{2 \pi} \int_{0}^{\pi} e^{j k r \cos \Lambda_{0}} S_{m n}^{(1)}\left(h, \cos \theta_{0}\right) e^{j m \phi_{0}} \sin \theta_{0} d \theta_{0} d \phi_{0} .
$$

In (1) $\Lambda_{0}$ (see Fig. 1) is the angle measured from the vector $\mathbf{r}$ to the propagation vector $\mathbf{k}_{0}$ (with modulus $k$ ) of an incoming plane wave propagating toward the direction $\left(\theta_{0}, \phi_{0}\right)$. The spheroidal angle function of the first kind $S_{m n}^{(1)}\left(h, \cos \theta_{0}\right)$ in the integrand of (1) is normally represented [11, pp. 16 and 22] by a series expansion of associated Legendre 
functions

$$
S_{m n}^{(1)}\left(h, \cos \theta_{0}\right)=\sum_{q=0,1}^{\infty} d_{q}^{m n}(h) P_{q+|m|}^{m}\left(\cos \theta_{0}\right)
$$

where the summation is over $q$ even if $n-m$ is even and is over $q$ odd if $n-m$ is odd; moreover, (2) is valid for both positive and negative values of $m$.

Substituting (2) into (1) and interchanging the order of summation and integration yields

$\Psi_{m n}^{(1)}(h ; \eta, \xi, \phi)=\sum_{q=0,1}^{\infty} d_{q}^{m n}(h) \frac{1}{4 \pi j^{n}} \int_{0}^{2 \pi} \int_{0}^{\pi} e^{j k r \cos \Lambda_{0}} P_{q+|m|}^{m}\left(\cos \theta_{0}\right) e^{j m \phi_{0}} \sin \theta_{0} d \theta_{0} d \phi_{0}$.

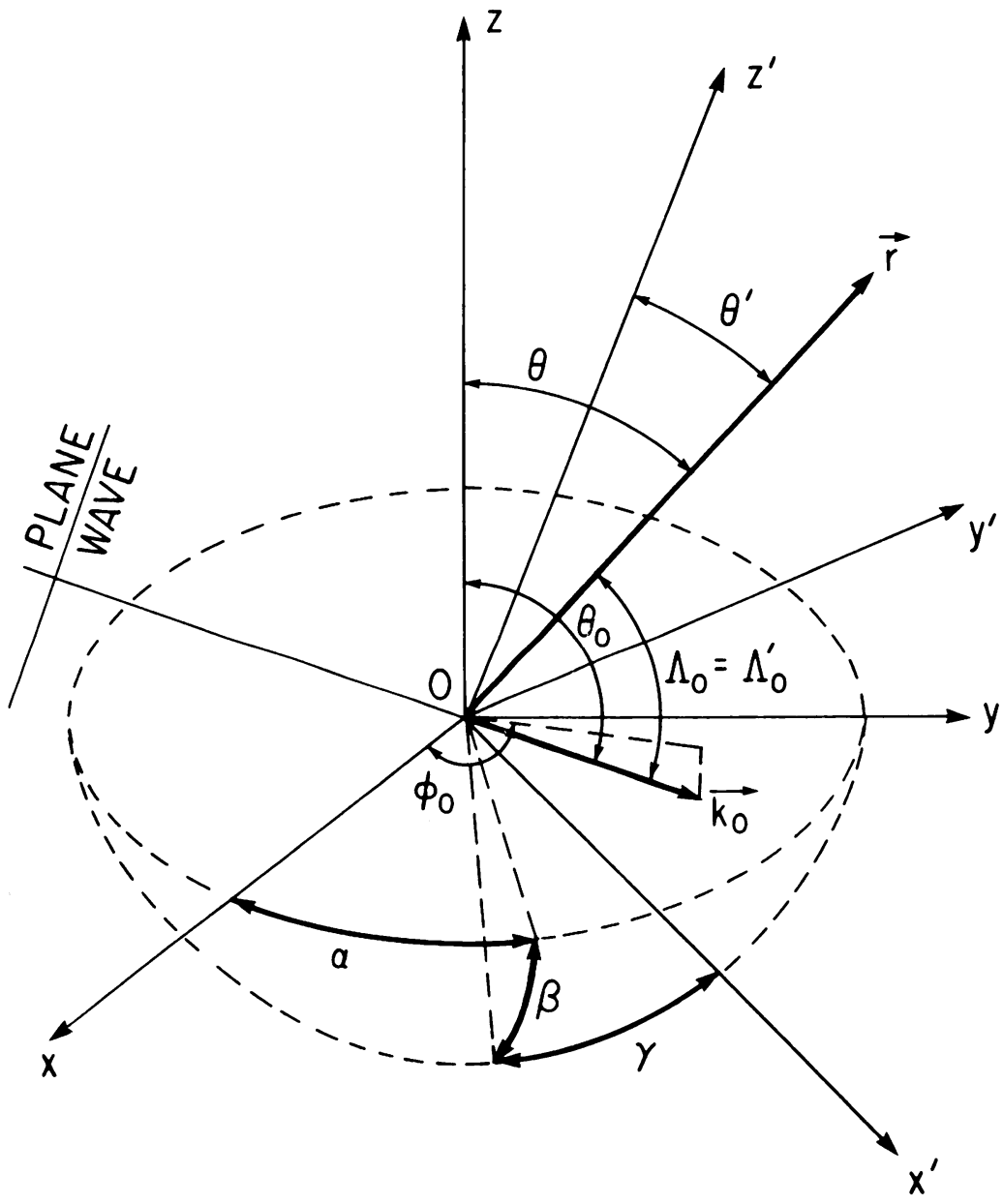

FIG. 1. The coordinate geometry of the two Cartesian systems $(x, y, z)$ and $\left(x^{\prime}, y^{\prime}, z^{\prime}\right)$ with a common origin $O$ and related by the Euler angles $(\alpha, \beta, \gamma)$. 
But if we use the well-known integral representation [13, p. 410; 11, p. 48, Eq. 5.3.7] of the spherical scalar wave function

$$
P_{n}^{m}(\cos \theta) j_{n}(k r) e^{j m \phi}=\frac{1}{4 \pi j^{n}} \int_{0}^{2 \pi} \int_{0}^{\pi} e^{j k r \cos \Lambda_{0}} P_{n}^{m}\left(\cos \theta_{0}\right) e^{j m \phi_{0}} \sin \theta_{0} d \theta_{0} d \phi_{0},
$$

then $(3)$ reduces to

$$
\Psi_{m n}^{(1)}(h ; \eta, \xi, \phi)=\sum_{q=0,1}^{\infty} j^{q+|m|-n} d_{q}^{m n}(h) P_{q+|m|}^{m}(\cos \theta) j_{q+|m|}(k r) e^{j m \phi} .
$$

Moreover, if $s=q+|m|$, then (5) becomes, after suppressing the $\phi$-dependence,

$$
S_{m n}^{(1)}(h, \eta) R_{m n}^{(1)}(h, \xi)=\sum_{s=|m| \cdot|m|+1}^{\infty} j^{s-n} d_{s-|m|}^{m n}(h) P_{s}^{m}(\cos \theta) j_{s}(k r) .
$$

This expression has previously been given by Dalmas and Deleuil [6, p. 1119] and differs from that provided by Flammer [11, p. 48, Eq. 5.3.8] by the factor $j^{s-n}$. It is noted that the $m s^{\text {th }}$ spherical wave function (scalar and of the first kind) is

$$
\Psi_{m s}^{(1)}(r, \theta, \phi)=j_{s}(k r) P_{s}^{m}(\cos \theta) e^{j m \phi} .
$$

Then (5) can be rewritten as

$$
\Psi_{m n}^{(1)}(h ; \eta, \xi, \phi)=\sum_{s=|m| .|m|+1}^{\infty} j^{s-n} d_{s-|m|}^{m n}(h) \cdot \Psi_{m s}^{(1)}(r, \theta, \phi) .
$$

In (8), both the spheroidal coordinate system $(\eta, \xi, \phi)$ and the spherical system $(r, \theta, \phi)$ are based on the original unprimed $(x, y, z)$ Cartesian reference frame with the $z$-axis as the axis of symmetry.

If we now transform the unprimed system into the primed $\left(x^{\prime}, y^{\prime}, z^{\prime}\right)$ system by rotations through three Euler angles $(\alpha, \beta, \gamma)$, then as Stein has remarked [2, Appendix I], there is no change in the nature of the scalar radial function in (7) but the $m s^{\text {th }}$ unnormalized spherical harmonic can be represented in the new primed system as follows:

$$
P_{s}^{m}(\cos \theta) e^{j m \phi}=\sum_{\mu=-s}^{s} R_{\mu s}^{m s}(\alpha, \beta, \gamma) P_{s}^{\mu}\left(\cos \theta^{\prime}\right) e^{j \mu \phi^{\prime}}
$$

with

$$
R_{\mu s}^{m s}(\alpha, \beta, \gamma)=(-1)^{\mu-m}\left[\frac{N_{m s}}{N_{\mu s}}\right]^{1 / 2} e^{j \mu \alpha} d_{\mu m}^{(s)}(\beta) e^{j m \gamma}
$$

This coefficient is discussed in detail in the Appendix.

If (9) is used in (7), after which the result is substituted into (8), then with $r=r^{\prime}$ we obtain

$$
\Psi_{m n}^{(1)}(h ; \eta, \xi, \phi)=\sum_{s=|m|,|m|+1}^{\infty} j^{s-n} d_{s-|m|}^{m n}(h) \sum_{\mu=-s}^{s} R_{\mu s}^{m s}(\alpha, \beta, \gamma)^{\cdot} \Psi_{\mu s}^{(1)}\left(r^{\prime}, \theta^{\prime}, \phi^{\prime}\right),
$$

which is recognized as a series expansion of the spheroidal scalar wave function $\Psi_{m n}^{(1)}(h ; \eta, \xi, \phi)$ in terms of spherical wave functions ${ }^{\prime} \Psi_{\mu s}^{\prime(1)}\left(r^{\prime}, \theta^{\prime}, \phi^{\prime}\right)=j_{s}\left(k r^{\prime}\right) P_{s}^{\mu}\left(\cos \theta^{\prime}\right) e^{j \mu \phi^{\prime}}$ of the first kind expressed in the rotated (primed) spherical coordinate system.

Our final task is to relate $\Psi_{\mu s}^{\prime(1)}\left(r^{\prime}, \theta^{\prime}, \phi^{\prime}\right)$ to the set of spheroidal scalar wave functions $\Psi_{\mu \nu}^{\prime(1)}\left(h^{\prime} ; \eta^{\prime}, \xi^{\prime}, \phi^{\prime}\right)$ based on the same rotated frame $\left(x^{\prime}, y^{\prime}, z^{\prime}\right)$, with $z^{\prime}$ as the axis of symmetry. To this end we now consider the integral representation of the $\mu s^{\text {th }}$ spherical 
scalar wave function $[13$, p. $410 ; 11$, p. 48$]$ of the first kind:

$$
\cdot \Psi_{\mu s}^{\prime(1)}\left(r^{\prime}, \theta^{\prime}, \phi^{\prime}\right)=\frac{1}{4 \pi j^{s}} \int_{0}^{2 \pi} \int_{0}^{\pi} e^{j k r^{\prime} \cos \Lambda_{0}^{\prime}} P_{s}^{\mu}\left(\cos \theta_{0}^{\prime}\right) e^{j \mu \phi_{0}^{\prime}} \sin \theta_{0}^{\prime} d \theta_{0}^{\prime} d \phi_{0}^{\prime} .
$$

For the exponential factor of the integrand of (12), Sinha and MacPhie [5, p. 145] provide the following series expansion, including negative values of the index $m^{\prime}$ :

$$
e^{j k r \cos \Lambda_{0}^{\prime}}=2 \sum_{\nu=0}^{\infty} \sum_{m^{\prime}=-\nu}^{\nu} \frac{j^{\nu}}{N_{m^{\prime} \nu}\left(h^{\prime}\right)} S_{m^{\prime} \nu}^{(1)}\left(h^{\prime}, \cos \theta_{0}^{\prime}\right) e^{-j m^{\prime} \phi_{0}^{\prime}} \Psi_{m^{\prime} \nu}^{(1)}\left(h^{\prime} ; \eta^{\prime}, \xi^{\prime}, \phi^{\prime}\right),
$$

where $N_{m^{\prime} v}\left(h^{\prime}\right)$ is the normalization coefficient [11, p. 22] for $S_{m^{\prime} \nu}^{(1)}\left(h^{\prime}\right)$. If (13) is substituted into (12) and the integration on $\phi^{\prime}$ is carried out, then only if $m^{\prime}=\mu$ does the integral not vanish. Moreover, if $S_{m^{\prime} \nu}^{(1)}\left(h^{\prime}, \cos \theta_{0}^{\prime}\right)$ in (13) is replaced by its Legendre series expansion [as given in (2)], the orthogonality of the associated Legendre functions for $\left|\cos \theta_{0}^{\prime}\right|<1$ is such that (12) reduces to

$$
\Psi_{\mu s}^{\prime(1)}\left(r^{\prime}, \theta^{\prime}, \phi^{\prime}\right)=N_{\mu s} \sum_{\nu=|\mu|,|\mu|+1}^{\infty} \frac{j^{\nu-s}}{N_{\mu \nu}\left(h^{\prime}\right)} d_{s-|\mu|}^{\mu \nu}\left(h^{\prime}\right) \Psi_{\mu \nu}^{\prime(1)}\left(h^{\prime} ; \eta^{\prime}, \xi^{\prime}, \phi^{\prime}\right) .
$$

It is to be noted that the series expansion (14) has been previously established for positive values of $\mu$ only, by Flammer [11, p. 49] and by Dalmas and Deleuil [6, p. 1119] for the spheroidal scalar wave function of the third kind.

It remains only to substitute (14) into (11), and we obtain

$$
\Psi_{m n}^{(1)}(h ; \eta, \xi, \phi)=\sum_{\mu=-\infty}^{\infty} \sum_{\nu=|\mu|,|\mu|+1}^{\infty} \mathscr{R}_{\mu \nu}^{m n}\left(h, h^{\prime} ; \alpha, \beta, \gamma\right) \Psi_{\mu \nu}^{(1)}\left(h^{\prime} ; \eta^{\prime}, \xi^{\prime}, \phi^{\prime}\right),
$$

where

$$
\mathscr{R}_{\mu \nu}^{m n}\left(h, h^{\prime} ; \alpha, \beta, \gamma\right)=j^{\nu-m} \sum_{s=s_{0}, s_{0}+1}^{\infty} \frac{N_{\mu s}}{N_{\mu \nu}\left(h^{\prime}\right)} d_{s-|m|}^{m n}(h) d_{s-|\mu|}^{\mu \nu}\left(h^{\prime}\right) R_{\mu s}^{m s}(\alpha, \beta, \gamma) .
$$

In the expression for $\mathscr{R}_{\mu \nu}^{m n}$ occurs the product $d_{s-|m|}^{m n}(h) d_{s-|\mu|}^{\mu \nu}\left(h^{\prime}\right)$; consequently, $\mathscr{R}_{\mu \nu}^{m n}$ does not vanish if and only if $n$ and $\nu$ have the same parity. In addition, the lower limit $s_{0}$ of $s$ in the summation is given by $|m|$ or $|\mu|$, whichever is greater, and moreover $s_{0}$ becomes $s_{0}+1$ if $\left|n+\nu-s_{0}\right|$ is odd.

The above analysis has focused on spheroidal scalar wave functions of the first kind whose radial behavior is of the standing wave type, expandable in terms of Bessel functions of the first kind $j_{m+r}(h \xi)[11$, p. 31]. If we now turn to the travelling wave type of spheroidal wave functions such as $\Psi_{m n}^{(i)}(h ; \eta, \xi, \phi)$ with $i=3,4$, whose variations with $\xi$ can be represented by a series combination of Hankel functions $h_{m+r}^{(i)}(h \xi)$ with $i=1,2$, then except for a change of limits for the integral representations (from $0 \leqslant \theta \leqslant \pi$ to $\left.0 \leqslant \theta \leqslant \frac{\pi}{2} \pm j \infty\right)$, the analysis is formally the same. In view of this fact, we recall that the series expansions (6) and (14), transforming spheroidal wave functions into spherical ones and conversely, have been previously established for $i=3[6, \mathrm{p}$. 1119]. The case $i=4$, corresponding to the use of $e^{j \omega t}$ instead of $e^{-j \omega t}$, leads to similar expansions. Consequently, (15) can be generalized to

$$
\Psi_{m n}^{(i)}(h ; \eta, \xi, \phi)=\sum_{\mu=-\infty}^{\infty} \sum_{\nu=|\mu|,|\mu|+1}^{\infty} \mathscr{R}_{\mu \nu}^{m n}\left(h, h^{\prime} ; \alpha, \beta, \gamma\right) \Psi_{\mu \nu}^{(i)}\left(h^{\prime} ; \eta^{\prime}, \xi^{\prime}, \phi^{\prime}\right) \quad(i=1,2,3,4) .
$$


It is interesting to note that, contrary to translational addition theorems [5-7] for spheroidal scalar wave functions, in the present rotational addition theorem there is never any change of the index $i$ from one side of (17) to the other.

3. Rotational-translational addition theorems for scalar spheroidal wave functions. In the interest of generality and in anticipation of the problem of several spheroidal scatterers with random positions and orientations, we now consider the case of $N$ spheroidal coordinate systems whose local origins $O_{q}$ are located at positions $\mathbf{d}_{q}(q=1,2, \ldots, N)$ relative to the global origin $O$, as illustrated in Fig. 2. The coordinate frame $\left(x_{q}, y_{q}, z_{q}\right)$ is obtained from the global Cartesian reference frame $(x, y, z)$ by rotations through the Euler angles $\left(\alpha_{q}, \beta_{q}, \gamma_{q}\right)$.

Our purpose is to deduce an addition formula which represents the $m n^{\text {th }}$ scalar spheroidal wave function $\Psi_{m n}^{(i)}\left(h_{q} ; \eta_{q}, \xi_{q}, \phi_{q}\right)$ in the $q^{\text {th }}$ coordinate system in terms of a series expansion of spheroidal wave functions $\Psi_{\mu \nu}^{(j)}\left(h_{r} ; \eta_{r}, \xi_{r}, \phi_{r}\right)$ in the $r^{\text {th }}$ system centered at $\mathbf{d}_{r}$ and with a local Cartesian frame $\left(x_{r}, y_{r}, z_{r}\right)$. As in the case of simple rotation (Sec. 2), we again begin with the integral representation

$$
\Psi_{m n}^{(1)}\left(h_{q} ; \mathbf{r}_{q}\right)=\frac{1}{4 \pi j^{n}} \int_{0}^{2 \pi} \int_{0}^{\pi} e^{j k r_{q} \cos \Lambda_{4}} S_{m n}^{(1)}\left(h_{q}, \cos \theta_{0 q}\right) e^{j m \phi_{0 q}} \sin \theta_{0 q} d \theta_{0 q} d \phi_{0 q},
$$

where $\mathbf{r}_{q}$ indicates the coordinate triad $\left(\eta_{q}, \xi_{q}, \phi_{q}\right)$ and $\Lambda_{q}$ is shown in Fig. 2. A series of substitutions identical to those that led from (1) to (8) permits us to represent $\Psi_{m n}^{(1)}\left(h_{q} ; \mathbf{r}_{q}\right)$ as follows:

$$
\Psi_{m n}^{(1)}\left(h_{q} ; \mathbf{r}_{q}\right)=\sum_{s=|m| .|m|+1}^{\infty} j^{s-n} d_{s-|m|}^{m n}\left(h_{q}\right) \cdot \Psi_{m s}^{(1)}\left(r_{q}, \theta_{q}, \phi_{q}\right),
$$

where $\Psi_{m s}^{(1)}\left(r_{q}, \theta_{q}, \phi_{q}\right)$ is the $m s^{\text {th }}$ spherical wave function in the $q^{\text {th }}$ spherical $\left(r_{q}, \theta_{q}, \phi_{q}\right)$ coordinate frame, based of course on the $q^{\text {th }}$ Cartesian frame $\left(x_{q}, y_{q}, z_{q}\right)$.

We next rotate the $q^{\text {th }}$ Cartesian frame into alignment (see Fig. 2) with the $r^{\text {th }}$ Cartesian frame $\left(x_{r}, y_{r}, z_{r}\right)$. In relation to the global frame $(x, y, z)$, the $q^{\text {th }}$ and $r^{\text {th }}$ frames each have a triad of associated Euler angles, namely $\left(\alpha_{q}, \beta_{q}, \gamma_{q}\right)$ and $\left(\alpha_{r}, \beta_{r}, \gamma_{r}\right)$.

Then, as in the case of simple rotation, when (8) was transformed into (11), we can rewrite (19) as

$$
\Psi_{m n}^{(1)}\left(h_{q} ; \mathbf{r}_{q}\right)=\sum_{s=|m|,|m|+1}^{\infty} j^{s-n} d_{s-|m|}^{m n}\left(h_{q}\right) \sum_{\bar{\mu}=-s}^{s} R_{\bar{\mu} s}^{m s}\left(\alpha_{q r}, \beta_{q r}, \gamma_{q r}\right) \cdot \Psi_{\bar{\mu} s}^{(1)}\left(r_{q}, \theta_{q r}, \phi_{q r}\right),
$$

in which $r_{q}$ remains $r_{q}$, the radial distance of $M$ (see Fig. 2) from the $q$ th origin $O_{q}$ located at $\mathbf{d}_{q}$; however, $\theta_{q}$ becomes $\theta_{q r}$ and $\phi_{q}$ becomes $\phi_{q r}$. The $q r^{\text {th }}$ and $r^{\text {th }}$ Cartesian coordinate frames are now aligned and separated by the displacement vector $\mathbf{d}_{q r}=\mathbf{d}_{r}-\mathbf{d}_{q}$.

In (20) we note that $\Psi_{\bar{\mu} s}^{(1)}\left(\mathbf{r}_{q r}\right)$ is the spherical wave function in the rotated reference frame. Cruzan [3, Appendix B] has given a translational addition theorem for spherical scalar wave functions and Flammer [11, p. 49] a theorem which allows us to convert spherical scalar wave functions into spheroidal ones (without translation and rotation). The use of the first theorem for the translation $O_{q} \rightarrow O_{r}$ gives us the following series for 
$\cdot \Psi_{\bar{\mu} s}^{(1)}:$

$$
\Psi_{\bar{\mu} s}^{(1)}\left(r_{q}, \theta_{q r}, \phi_{q r}\right)=\sum_{l=0}^{\infty} \sum_{\mu=-l}^{l}{ }^{(1)} a_{\mu l}^{\bar{\mu} s}\left(d_{q r}, \bar{\theta}_{q r}, \bar{\phi}_{q r}\right) \cdot \Psi_{\mu l}^{(1)}\left(r_{r}, \theta_{r}, \phi_{r}\right)
$$

with

$$
{ }^{(1)} a_{\mu l}^{\bar{\mu} s}\left(d_{q r}, \bar{\theta}_{q r}, \bar{\phi}_{q r}\right)=(-1)^{\mu} \sum_{p} j^{l+p-s}(2 l+1) a(\bar{\mu}, s|-\mu, l| p)^{\cdot} \Psi_{\bar{\mu}-\mu, p}^{(1)}\left(d_{q r}, \bar{\theta}_{q r}, \bar{\phi}_{q r}\right)
$$

where $\left(d_{q r}, \bar{\theta}_{q r}, \bar{\phi}_{q r}\right)$ are the spherical coordinates of the displacement vector $\mathbf{d}_{q r}=\mathbf{d}_{q}-\mathbf{d}_{r}$ (see Fig. 2) in the $q r^{\text {th }}$ coordinate frame. By using the second theorem due to Flammer

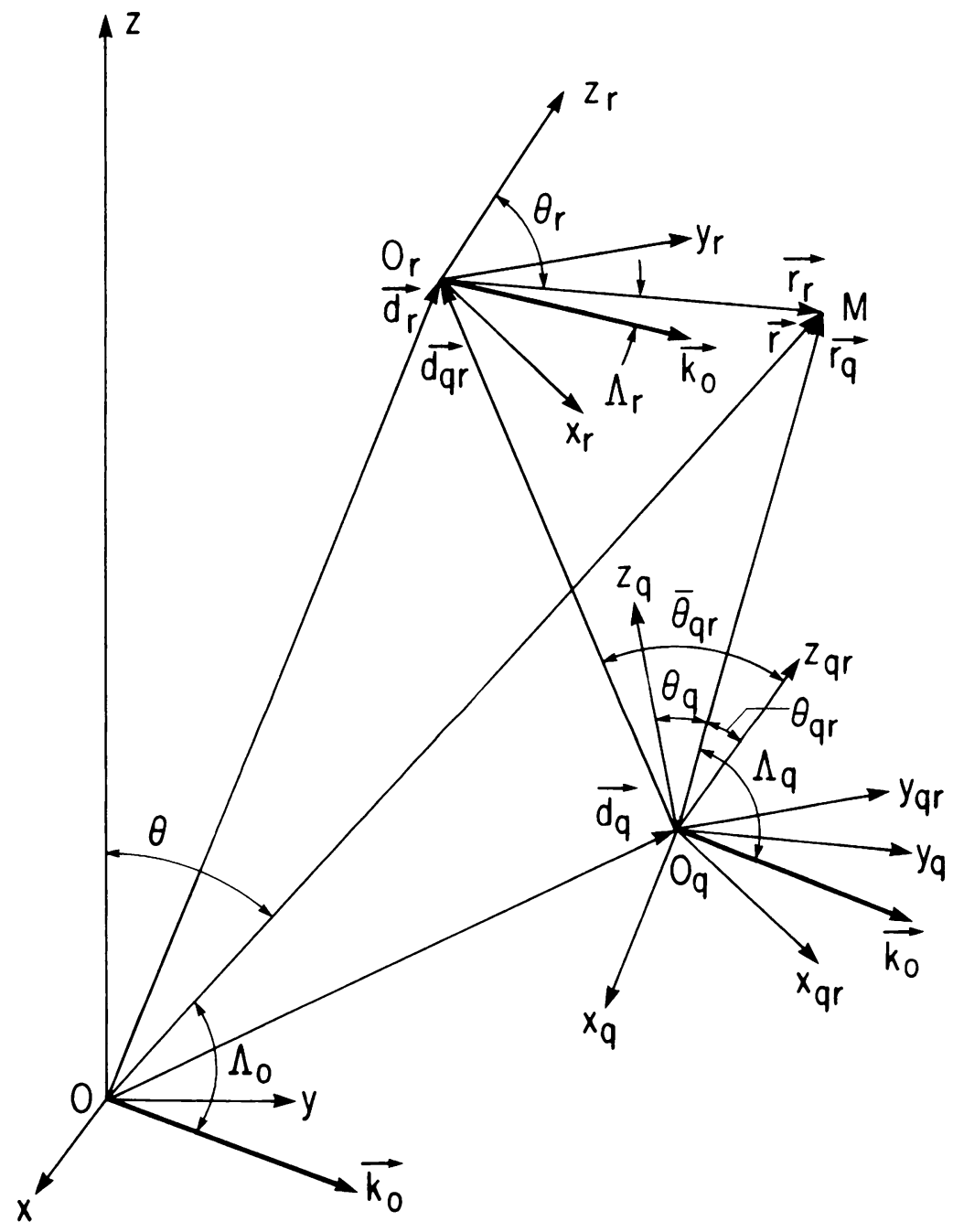

FIG. 2. The coordinate geometry of the two Cartesian systems $\left(x_{q}, y_{q}, z_{q}\right)$ and $\left(x_{r}, y_{r}, z_{r}\right)$ with arbitrary positions and orientations relative to the global system $(x, y, z)$. 
cited above, (21) becomes

$$
\cdot \Psi_{\bar{\mu} s}^{(1)}\left(r_{q}, \theta_{q r}, \phi_{q r}\right)=\sum_{\nu=0}^{\infty} \sum_{\mu=-\nu}^{\nu}{ }^{(1)} A_{\mu \nu}^{\mu s}\left(\mathbf{d}_{q r}\right) \Psi_{\mu \nu}^{(1)}\left(h_{r} ; \eta_{r}, \xi_{r}, \phi_{r}\right),
$$

with

$$
\begin{aligned}
{ }^{(1)} A_{\mu \nu}^{\bar{\mu} s}\left(\mathbf{d}_{q r}\right)= & \frac{(-1)^{\mu}}{N_{\mu \nu}\left(h_{r}\right)} \sum_{l=|\mu| \cdot|\mu|+1}^{\infty}(2 l+1)^{\cdot} N_{\mu l} d_{l-|\mu|}^{\mu \nu}\left(h_{r}\right) \sum_{p} j^{p+\nu-s} \\
& \cdot a(\bar{\mu}, s|-\mu, l| p)^{\cdot} \Psi_{\bar{\mu}-\mu, p}^{(1)}\left(d_{q r}, \bar{\theta}_{q r}, \bar{\phi}_{q r}\right) .
\end{aligned}
$$

The final step is to use (23) in (20); we find that

$$
\Psi_{m n}^{(1)}\left(h_{q} ; \mathbf{r}_{q}\right)=\sum_{\nu=0}^{\infty} \sum_{\mu=-\nu}^{\nu}{ }^{(1)} Q_{m n ; \mu \nu}^{q r}\left(\alpha_{q r}, \beta_{q r}, \gamma_{q r} ; \mathbf{d}_{q r}\right) \Psi_{\mu \nu}^{(1)}\left(h_{r} ; \mathbf{r}_{r}\right) .
$$

In (25), the ${ }^{(1)} Q_{m n ; \mu \nu}^{q r}$ coefficients are the rotational-translational expansion coefficients of the first kind that we have been seeking. It is straightforward to show that ${ }^{(1)} Q_{m n: \mu \nu}^{q r}$ can be written as follows:

$$
\begin{aligned}
& { }^{(1)} Q_{m n ; \mu \nu}^{q r}\left(\alpha_{q r}, \beta_{q r}, \gamma_{q r}, \mathbf{d}_{q r}\right)=\sum_{s=|m| \cdot|m|+1}^{\infty} d_{s-|m|}^{m n}\left(h_{q}\right) \\
& \cdot \sum_{\bar{\mu}=-s}^{s} R_{\bar{\mu} s}^{m s}\left(\alpha_{q r}, \beta_{q r}, \gamma_{q r}\right) \sum_{l=|\mu| \cdot|\mu|+1}^{\infty}{ }^{(1)} a_{\mu l}^{\bar{\mu} s}\left(\mathbf{d}_{q r}\right) j^{s-n+\nu-1} \frac{N_{\mu l}}{N_{\mu \nu}\left(h_{r}\right)} d_{l-|\mu|}^{\mu \nu}\left(h_{r}\right) .
\end{aligned}
$$

In (26), if $n-|m|$ is even (odd) the lower limit of the summation on $s$ is $|m|(|m|+1)$; a similar rule governs the lower limit of the summation on $l$. As can be seen, (25) transforms spheroidal scalar wave functions of the first kind expressed in the $q^{\text {th }}$ coordinate system into a series expansion of spheroidal scalar wave functions of the first kind expressed in the $r^{\text {th }}$ coordinate system.

In the same way, scalar rotational-translational addition theorems for the wave functions of the third and fourth kinds can be obtained, but the two cases, $r_{r} \leqslant d_{q r}$ and $r_{r} \geqslant d_{q r}$, must be distinguished during the translation operation, as is done for the scalar addition theorems of spherical wave functions given by Cruzan [3] and used here.

We first limit our attention to the case $r_{r} \leqslant d_{q r}$, a case very useful in studies of multiple scattering of scalar or vector spheroidal waves. In the same way as for $\Psi_{m n}^{(1)}\left(h_{q} ; \mathbf{r}_{q}\right)$, it is easy to deduce a rotational-translational addition theorem for $\psi_{m n}^{(i)}\left(h_{q} ; \mathbf{r}_{q}\right)$ when $i=2,3,4$ by using, in place of ${ }^{(1)} a_{\mu l}^{\bar{\mu} s}$, the following coefficient:

$$
{ }^{(i)} a_{\mu l}^{\bar{\mu} s}\left(d_{q r}, \bar{\theta}_{q r}, \bar{\phi}_{q r}\right)=(-1)^{\mu} \sum_{p} j^{l+p-s}(2 l+1) a(\bar{\mu}, s|-\mu, l| p) \cdot \Psi_{\bar{\mu}-\mu, p}^{(i)}\left(d_{q r}, \bar{\theta}_{q r}, \bar{\phi}_{q r}\right),
$$

obtained from the form $B-1$ of the addition theorem for spherical scalar wave functions, given in [3]. In addition, we recall that the series expansions (19) and (13) are also valid for $i=3$. Taking into account the definitions of the spherical Hankel functions of the first and second kinds, it is evident that they are also valid for $i=4$ and $i=2$. Therefore, we 
can write

$$
\Psi_{m n}^{(i)}\left(h_{q} ; \mathbf{r}_{q}\right)=\sum_{\nu=0}^{\infty} \sum_{\mu=-\nu}^{\nu}{ }^{(i)} Q_{m n ; \mu \nu}^{q r}\left(\alpha_{q r}, \beta_{q r}, \gamma_{q r} ; \mathbf{d}_{q r}\right) \Psi_{\mu \nu}^{(1)}\left(h_{r} ; \mathbf{r}_{r}\right) \text {, }
$$

valid only if $r_{r} \leqslant d_{q r}$. The formula for ${ }^{(i)} Q_{m n ; \mu \nu}^{q r}$ is deduced from (26) by replacing ${ }^{(1)} a_{\mu l}^{\bar{\mu} s}$ with ${ }^{(i)} a_{\mu l}^{\bar{\mu} s}$ given by (27), and the choice of the parity of the indices $s$ and $l$ obeys the same rules as for ${ }^{(1)} Q_{m n: \mu \nu}^{q r}$.

The case $r_{r} \geqslant d_{q r}$ can be treated if we consider the form $B$-2 of the addition theorem of spherical scalar wave functions [3, p. 40]. In the present notation, $B-2$ becomes

$$
\cdot \Psi_{\bar{\mu} s}^{(i)}\left(r_{q}, \theta_{q r}, \phi_{q r}\right)=\sum_{l=0}^{\infty} \sum_{\tilde{\mu}=-l}^{l} \sum_{p}^{(1)} b_{\tilde{\mu}, l, p}^{\bar{\mu} s}\left(\mathbf{d}_{q r}\right) \cdot \Psi_{\bar{\mu}-\tilde{\mu}, p}^{(i)}\left(r_{r}, \theta_{r}, \phi_{r}\right),
$$

with

$$
{ }^{(1)} b_{\tilde{\mu}, l, p}^{\bar{\mu} s}\left(\mathbf{d}_{q r}\right)=(-1)^{\tilde{\mu}} j^{l+p-s}(2 l+1) a(\bar{\mu}, s|-\tilde{\mu}, l| p) \cdot \Psi_{\tilde{\mu} l}^{(1)}\left(\mathbf{d}_{q r}\right) .
$$

In (29) the index $p$ is such that $p=p_{0}, p_{0}+2, p_{0}+4, \ldots, l+s$, with $p_{0}=\max [|l-s|$, $|\bar{\mu}-\mu|]$. However, it turns out that the series on the index $l$ in (29), with $p$ and $s$ held constant, is of a finite number of terms with $l=l_{0}, l_{0}+2, l_{0}+4, \ldots, p+s$, where $l_{0}=\max [|p-s|,|\tilde{\mu}|]$. Moreover, it will prove to be very useful to let $\bar{\mu}-\tilde{\mu}=\mu$. With these changes of summation indices and summation limits, it is possible to rewrite (29) as follows:

$$
\cdot \Psi_{\bar{\mu} s}^{(i)}\left(r_{q}, \theta_{q r}, \phi_{q r}\right)=\sum_{\mu=-\infty}^{\infty} \sum_{p=|\mu|}^{\infty} \sum_{l=l_{0}, l_{0}+1}^{p+s}{ }^{(1)} b_{\bar{\mu}-\mu, l, p}^{\bar{\mu} s}\left(\mathbf{d}_{q r}\right) \cdot \Psi_{\mu p}^{(i)}\left(\mathbf{r}_{r}\right),
$$

which simplifies to

$$
\cdot \Psi_{\bar{\mu} s}^{(i)}\left(r_{q}, \theta_{q r}, \phi_{q r}\right)=\sum_{\mu=-\infty}^{\infty} \sum_{p=|\mu|}^{\infty}{ }^{(1)} b_{\mu p}^{\bar{\mu} s}\left(\mathbf{d}_{q r}\right) \cdot \Psi_{\mu p}^{(i)}\left(\mathbf{r}_{r}\right)
$$

where

$$
{ }^{(1)} b_{\mu p}^{\bar{\mu} s}\left(\mathbf{d}_{q r}\right)=\sum_{l=l_{0}, l_{0}+1}^{p+s}{ }^{(1)} b_{\bar{\mu}-\mu, l, p}^{\bar{\mu} s}\left(\mathbf{d}_{q r}\right) .
$$

If, in (32), ${ }^{\top} \Psi_{\mu p}^{(i)}\left(\mathbf{r}_{r}\right)$ is replaced by the series expansion of spheroidal wave functions as given by (14), which is valid for $i=1,2,3,4$, and if one substitutes the resulting expansion for $\Psi_{\bar{\mu} s}^{(i)}\left(r_{q}, \theta_{q r}, \phi_{q r}\right)$ into (20), where 1 has been replaced by $i$, then after rearrangement of the order of summation we find that

$$
\Psi_{m n}^{(i)}\left(h_{q} ; \mathbf{r}_{q}\right)=\sum_{\mu=-\infty}^{\infty} \sum_{\nu=|\mu|}^{\infty}{ }^{(1)} P_{m n ; \mu \nu}^{q r}\left(\alpha_{q r}, \beta_{q r}, \gamma_{q r} ; \mathbf{d}_{q r}\right) \Psi_{\mu \nu}^{(i)}\left(h_{r} ; \mathbf{r}_{r}\right),
$$

where

$$
\begin{aligned}
{ }^{(1)} P_{m n ; \mu \nu}^{q r}= & \sum_{s=|m|,|m|+1}^{\infty} d_{s-|m|}^{m n}\left(h_{q}\right) \sum_{\bar{\mu}=-s}^{s} R_{\bar{\mu} s}^{m s}\left(\alpha_{q r}, \beta_{q r}, \gamma_{q r}\right) \\
& \sum_{p=|\mu|,|\mu|+1}^{\infty}{ }^{(1)} b_{\mu p}^{\ddot{\mu} s}\left(\mathbf{d}_{q r}\right) \cdot N_{\mu p} \frac{j^{\nu-p}}{N_{\mu \nu}\left(h_{r}\right)} d_{p-|\mu|}^{\mu \nu}\left(h_{r}\right) .
\end{aligned}
$$


In (35) if $n-|m|$ is even the lower limit on $s$ is $|m|$; if $n-|m|$ is odd the lower limit is $|m|+1$. An identical rule governs the lower limits on $p$.

4. Matrix representation of the rotational-translational operator. The rather complicated formulas for the expansion coefficients for the rotational-translational theorems [see (26) and (35)] can be succinctly represented in terms of certain matrix products. We first reorder the doubly-indexed set of eigenfunctions $\Psi_{m n}^{(1)}\left(h_{q} ; \mathbf{r}_{q}\right)$ as a singly-indexed set such that the $k^{\text {th }}$ element in the latter is related to the $m n^{\text {th }}$ element in the former by the formula

$$
k=\hat{m} N_{q}+n+1
$$

where

$$
\hat{m}= \begin{cases}2 m-1, & m>0, \\ 2|m|, & m \leqslant 0,\end{cases}
$$

and $|m| \leqslant n \leqslant N_{q}$; consequently, $k=1,2, \ldots,\left(N_{q}+1\right)^{2}$, where $N_{q}$ is the upper limit on $n$, dictated by computational and/or physical restraints.

In a similar fashion we reorder the doubly-indexed eigenfunctions $\Psi_{\mu \nu}^{(1)}\left(h_{r} ; \mathbf{r}_{r}\right)$ so that

$$
j=\hat{\mu} N_{r}+\nu+1
$$

where

$$
\hat{\mu}= \begin{cases}2 \mu-1, & \mu>0, \\ 2|\mu|, & \mu \leqslant 0,\end{cases}
$$

and $|\mu| \leqslant \nu \leqslant N_{r}$; as a result, $j=1,2, \ldots,\left(N_{r}+1\right)^{2}$. We note that, in general, $N_{q} \neq N_{r}$.

It can then be shown that the rotational-translational matrix $\left[{ }^{(1)} Q^{q r}\right]$, whose elements are ${ }^{(1)} Q_{m n ; \mu \nu}^{q r}$ or ${ }^{(1)} Q_{k j}^{q r}$ coefficients, as given by (26), can be obtained by means of the following matrix product:

$$
\left[{ }^{(1)} Q^{q r}\right]=\left[D^{r}\right]\left[{ }^{(1)} T\right][R]\left[\Delta^{q}\right] .
$$

In (36), $\left[D^{r}\right]$ is a matrix of $\left(N_{r}+1\right)^{2}$ rows whose element in the $\mu \nu^{\text {th }}$ or $j^{\text {th }}$ row and $\tilde{\mu} l^{\text {th }}$ or $k_{1}^{\text {th }}$ column is

$$
D_{\mu \nu}^{\tilde{\mu} l}=D_{j k_{1}}^{r}=\delta_{\tilde{\mu} \mu} \cdot N_{\tilde{\mu} l} \frac{j^{\mu-l}}{N_{\mu \nu}\left(h_{r}\right)} d_{l-|\mu|}^{\mu \nu}\left(h_{r}\right),
$$

where $\delta_{\tilde{\mu} \mu}$ is the Kronecker delta. The number of columns $K_{r}$ depends on the desired accuracy and on the parameter $h_{r}$, with an increase in $h_{r}=2 \pi F_{r} / \lambda$ dictating a proportionate increase of $K_{r}$. The matrix $\left[{ }^{(1)} T\right]$ in (36) is the spherical-spherical translation matrix with $K_{r}$ rows and $J_{q}$ columns, where $J_{q}$ is the number of rows in $\left[\Delta^{q}\right]$, a number which is proportional to $h_{q}=2 \pi F_{q} / \lambda$; the element in the $k_{1}^{\text {th }}$ or $\tilde{\mu} l^{\text {th }}$ row and $j_{1}^{\text {th }}$ or $\bar{\mu} \bar{s}^{\text {th }}$ column of $\left[{ }^{(1)} T\right]$ is

$$
{ }^{(1)} T_{k_{\mathrm{L}} j_{1}}={ }^{(1)} a_{\tilde{\mu} l}^{\bar{\mu} \hat{s}},
$$

where ${ }^{(1)} a_{\tilde{\mu} /}^{\bar{\mu} \bar{s}}$ is given by (22). The spherical-spherical rotation matrix $[R]$ is square with $K_{r}^{2}$ elements; in the $j_{1}^{\text {th }}$ row and $k_{2}^{\text {th }}$ or $\tilde{m} s^{\text {th }}$ column we find

$$
R_{j_{1} k_{2}}=R_{\bar{\mu} \bar{s}}^{\tilde{m} s}=\delta_{s \bar{s}} R_{\bar{\mu} s}^{\tilde{m} s}
$$


where $R_{\bar{\mu} s}^{\tilde{m} s}$ is specified by (10). The final matrix in (36) is $\left[\Delta^{q}\right]$ with $J_{q}$ rows and $\left(N_{q}+1\right)^{2}$ columns. In the $k_{2}^{\text {th }}$ row and $k^{\text {th }}$ column the matrix element is

$$
\Delta_{k_{2} k}^{q}=\delta_{m \tilde{m}} j^{s-n} d_{s-|\tilde{m}|}^{m m}\left(h_{q}\right) .
$$

Matrix product representations analogous to (36) can also be obtained for the more general cases where $i$ replaces 1 [as in (28)] and where $r_{r} \geqslant d_{q r}$ [as in (34)]. Thus the coefficient matrix whose elements are the coefficients in (28) is given by

$$
\left[{ }^{(i)} Q^{q r}\right]=\left[D^{r}\right]\left[{ }^{(i)} T\right][R]\left[\Delta^{q}\right] .
$$

In (41) the matrix elements of $\left[{ }^{(i)} T\right]$ are simple generalizations of the elements of $\left[{ }^{(1)} T\right]$, as given by (38) and (22). For the case of $r_{r} \geqslant d_{q r}$ we can write

$$
\left[{ }^{(1)} P^{q r}\right]=\left[D^{r}\right]\left[{ }^{(1)} \tilde{T}\right][R]\left[\Delta^{q}\right],
$$

where the element in the $k_{1}^{\text {th }}$ row and $j_{1}^{\text {th }}$ column of $\left[{ }^{(1)} \tilde{T}\right]$ is

$$
{ }^{(1)} \tilde{T}_{k_{1} j_{1}}={ }^{(1)} b_{\dot{\mu} p}^{\bar{\mu} \bar{s}}\left(\mathbf{d}_{q r}\right)
$$

[see (33)]. All other matrices are the same as those in (36) and are of a quasi-diagonal nature; thus the elements of $\left[D^{r}\right]$ and $\left[D^{q}\right]$ vanish when $\tilde{\mu} \neq \mu$ and $\tilde{m} \neq m$, respectively, whereas the elements of $[R]$ vanish when $\bar{s} \neq s$. This is, of course, very useful in reducing the time of computation in practical applications.

5. Extension to the case of vector wave functions. Both spherical and spheroidal vector wave functions can be generated from the scalar wave functions [13, p. 414; 11, p. 69] by the simple vector operations

$$
\begin{aligned}
\mathbf{M}_{m n}^{(a)} & =\nabla \Psi_{m n} \times \mathbf{a}, \\
k \mathbf{N}_{m n}^{(a)} & =\nabla \times \mathbf{M}_{m n}^{(a)},
\end{aligned}
$$

where $\mathbf{a}$ is a constant vector or radius vector.

For the case of simple rigid body rotation and spherical wave functions, Stein [2, p. 17] has shown that it is "extremely easy" to describe the addition theorems for $\mathbf{M}_{m n}^{(a)}$ and $\mathbf{N}_{m n}^{(a)}$ when a is a radius vector. On the other hand, Sinha and MacPhie [5, p. 152] have demonstrated that for simple rigid body translation the addition theorems for spheroidal vector wave functions are easily deduced if $\mathbf{a}$ is any one of the three Cartesian unit vectors, $\hat{\mathbf{x}}, \hat{\mathbf{y}}, \hat{\mathbf{z}}$. The present problem, which involves both rotation and translation, appears to lack a very simple solution for the vector case. Nevertheless, it is our intent to seek a solution, which is indispensable for the rigorous analysis of the radiation and/or scattering of electromagnetic waves from spheroidal bodies having arbitrary positions and orientations.

Appendix. Let $R(\alpha, \beta, \gamma)$ be the operator which represents the finite rotation under present consideration and let $D_{m^{\prime} m}^{(l)}(\alpha, \beta, \gamma)$ be its matrix representation. This rotation operator is the product of three operators $R_{\gamma} R_{\beta} R_{\alpha}$ with $R_{\alpha}$ operating on the wave function first. It can be shown $[12$, p. $8 ; 14$, p. $51 ; 15$, p. 20$]$ that these rotations may all be carried out in the same coordinate system if the order of rotations is inverted. In this way we obtain, according to the notation of Edmonds [12]

$$
D_{m^{\prime} m}^{(l)}(\alpha, \beta, \gamma)=e^{j m^{\prime} \alpha} d_{m^{\prime} m}^{(l)}(\beta) e^{j m \gamma} .
$$


If we now consider the $l m^{\text {th }}$ normalized spherical harmonic [12, p. $24 ; 14$, p. 240]

$$
Y_{l m}(\theta, \phi)=(-1)^{m}\left[\frac{(2 l+1)(l-m) !}{4 \pi(l+m) !}\right]^{1 / 2} P_{l}^{m}(\cos \theta) e^{j m \phi},
$$

which is related to the associated Legendre functions taken in Ferrer's notation, we have

$$
Y_{l m}(\theta, \phi)=\sum_{m^{\prime}=-l}^{l} D_{m^{\prime} m}^{(l)}(\alpha, \beta, \gamma) \gamma_{l m^{\prime}}\left(\theta^{\prime}, \phi^{\prime}\right) \text {. }
$$

In the literature, one can find various expressions for the elements $d_{m^{\prime} m}^{(l)}(\beta)$ of the rotation matrix $[12$, pp. $57-58 ; 14$, p. $52 ; 15$, p. 22$]$. In the present work, we select the following:

$$
d_{m_{m}}^{(l)}(\beta)=\left[\frac{\left(l+m^{\prime}\right) !\left(l-m^{\prime}\right) !}{(l+m) !(l-m) !}\right]^{1 / 2} P_{l-m^{\prime}}^{\left(m^{\prime}-m, m^{\prime}+m\right)}(\cos \beta)(\cos \beta / 2)^{m^{\prime}+m}(\sin \beta / 2)^{m^{\prime}-m}
$$

given by Edmonds in terms of the Jacobi polynomials $P_{n}^{(a, b)}(\cos \beta)$, which are used according to the definition of Szegö [16, vol. II, p. 167; 17, chapter 22].

Now, we can easily deduce the addition theorem for the unnormalized spherical harmonics under coordinate rotations. We obtain, in view of (A-2) and (A-3):

$$
P_{l}^{m}(\cos \theta) e^{j m \phi}=\sum_{m^{\prime}=-l}^{l}(-1)^{m^{\prime}-m}\left[\frac{(l+m) !\left(l-m^{\prime}\right) !}{(l-m) !\left(l+m^{\prime}\right) !}\right]^{1 / 2} D_{m^{\prime} m}^{(l)}(\alpha, \beta, \gamma) P_{l}^{m^{\prime}}\left(\cos \theta^{\prime}\right) e^{j m^{\prime} \phi^{\prime}} \text {. }
$$

If we introduce the normalization factor of the associated Legendre functions

$$
N_{m l}=\frac{2}{2 l+1} \frac{(l+m) !}{(l-m) !}
$$

then we write instead of (A-5)

$$
P_{l}^{m}(\cos \theta) e^{j m \phi}=\sum_{m^{\prime}=-l}^{l}(-1)^{m^{\prime}-m}\left[\frac{{ }^{\prime} N_{m l}}{N_{m^{\prime} l}}\right]^{1 / 2} e^{j m^{\prime} \alpha} d_{m^{\prime} m}^{(l)}(\beta) e^{j m \gamma} P_{l}^{m^{\prime}}\left(\cos \theta^{\prime}\right) e^{j m^{\prime} \phi},
$$

or more briefly:

$$
P^{m}(\cos \theta) e^{j m \phi}=\sum_{m^{\prime}=-l}^{l} R_{m^{\prime} l}^{m l}(\alpha, \beta, \gamma) P_{l}^{m^{\prime}}\left(\cos \theta^{\prime}\right) e^{j m^{\prime} \phi^{\prime}},
$$

with

$$
R_{m^{\prime} l}^{m l}(\alpha, \beta, \gamma)=(-1)^{m^{\prime}-m}\left[\frac{{ }^{\prime} N_{m l}}{N_{m^{\prime} l}}\right]^{1 / 2} e^{j m^{\prime} \alpha} d_{m^{\prime} m}^{(l)}(\beta) e^{j m \gamma} .
$$

In (A-5)-(A-8) the element $d_{m m}^{(l)}(\beta)$ can be evaluated by any of the expressions mentioned above, and, in particular, we can use expression (A-4), given in terms of Jacobi polynomials. In our opinion, this is the most suitable choice because the Jacobi polynomials are related by recurrence formulas, which can in turn generate recurrence formulas for 
the $d_{m^{\prime} m}^{(l)}(\beta)$. Two have been obtained: one by Altmann and Bradley [19, p. 196] and the other by Gel'Fand and Sapiro [18, p. 288]. Moreover, others which are more useful for programming have recently been developed by the present authors.

\section{REFERENCES}

[1] B. Friedman and J. Russek, Addition theorems for spherical waves, Quart. Appl. Math. 12, 13-23 (1954)

[2] S. Stein, Addition theorems for spherical wave functions, Quart. Appl. Math. 19, 15-24 (1961)

[3] O. Cruzan, Translational addition theorems for spherical vector wave functions, Quart. Appl. Math. 20, 33-40 (1962)

[4] J. Bruning and Y. T. Lo, Multiple scattering of EM waves by spheres, part I and part II, IEEE Trans. AP-19, $378-400(1971)$

[5] B. P. Sinha and R. H. MacPhie, Translational addition theorems for spheroidal scalar and vector wave functions, Quart. Appl. Math. 38, 143-158 (1980)

[6] J. Dalmas and R. Deleuil, Diffusion multiple des ondes électromagnétiques par des ellipsödes de révolution allongés, Opt. Acta 29,1117-1131 (1982)

[7] J. Dalmas and R. Deleuil, Translational addition theorems for prolate spheroidal vector wave functions $\mathbf{M}^{r}$ and $\mathbf{N}^{r}$, Quart. Appl. Math. 44, 213-222 (1986)

[8] B. P. Sinha and R. H. MacPhie, Electromagnetic plane wave scattering by a system of two parallel conducting prolate spheroids, IEEE Trans. AP-31, 294-304 (1983)

[9] J. Dalmas and R. Deleuil, Multiple scattering of electromagnetic waves from two infinitely conducting prolate spheroids which are centered in a plane perpendicular to their axes of revolution, Radio Sci. 20, 575-581 (1985)

[10] B. P. Sinha and R. H. MacPhie, Mutual admittance characteristics for two-element parallel prolate spheroidal antenna systems, IEEE Trans. AP-33, 1255-1263 (1985)

[11] C. Flammer, Spheroidal wave functions, Stanford Univ. Press, Stanford, Calif. (1957)

[12] A. R. Edmonds, Angular momentum in quantum mechanics, Princeton Univ. Press, Princeton, N. J. (1957)

[13] J. A. Stratton, Electromagnetic theory, McGraw-Hill, New York (1941)

[14] M. E. Rose, Elementary theory of angular momentum, Wiley, New York (1967)

[15] D. M. Brink and G. R. Satchler, Angular momentum, Clarendon, Oxford (1962)

[16] A. Erdelyi, Higher transcendental functions, McGraw-Hill, New York (1953)

[17] M. Abramowitz and I. Stegun, Handbook of mathematical functions, Dover, New York (1965)

[18] I. M. Gel'Fand and Z. Ya. Sapiro, Representations of the group theory of rotations of a 3-dimensional space and their applications, Ann. Math. Soc. Transl., Ser. 2, 2, 207-316 (1956)

[19] S. L. Altmann and C. J. Bradley, A note on the calculation of the matrix elements of the rotation group. Philos. Trans. Roy. Soc. London, Ser. A, 255, 193-198 (1963) 